\title{
Erratum to: Natural convection heat transfer of a viscous fluid in a vertical porous channel
}

\author{
F. Talay Akyildiz - Dennis A. Siginer • \\ K. Vajravelu • Robert A. Van Gorder
}

Published online: 5 March 2016

(C) Springer Science+Business Media Dordrecht 2016

\section{Erratum to: J Eng Math (2012) 74:61-71 DOI 10.1007/s10665-011-9489-x}

The original article was published with typographical errors in the nondimensionalization.

The corrected version of the nondimensionalization, the present address of the fourth author, and the present e-mail addresses of the fourth and the corresponding authors are given in the erratum. The Mathematics Subject Classification codes which are omitted in the original publication are also given below.

Mathematics Subject Classification 35K05 - 60H05 - 91G80

In Akyildiz et al. [1] we considered the ODE system (2.7) and (2.11) which takes the form

$k \frac{\mathrm{d}^{2} T}{\mathrm{~d} y^{2}}+\mu\left(\frac{\mathrm{d} u}{\mathrm{~d} y}\right)^{2}+Q^{\prime \prime}=0, \quad \frac{\mathrm{d}^{2} u}{\mathrm{~d} y^{2}}-\frac{1}{K} u+\frac{\beta_{0}}{\mu}\left(1+\frac{\beta_{1}}{\beta_{0}} \tau\right) \rho g \tau=0$,

governing natural convection heat transfer of a viscous fluid in a vertical porous channel. Here $\beta_{0}=(\partial \rho / \partial T)_{\mathrm{S}}$ and $\beta_{1}=\left(\partial^{2} \rho / \partial T^{2}\right)_{\mathrm{S}}$ are, respectively, the LDT and the QDT parameters, $\tau=T-T_{\mathrm{S}}$, and we take $\mathrm{d}\left(p-p_{\mathrm{S}}\right) / \mathrm{d} x=0$ (see, for details [2-4]). Note that $\beta_{0}, \beta_{1}$ can be given different functional forms, but in practice these values can be

The online version of the original article can be found under doi:10.1007/s10665-011-9489-x.

F. T. Akyildiz

Department of Mathematics, College of Arts \& Sciences, Petroleum Institute, P.O. Box 2533, Abu Dhabi, UAE

D. A. Siginer

Centro de Investigaciones en Creatividad y Educacion Superior, Universidad de Santiago de Chile, Santiago, Chile

K. Vajravelu $(\varangle)$

Department of Mathematics, University of Central Florida, Orlando, FL 32816, USA

e-mail: kuppalapalle.vajravelu@ucf.edu

\section{R. A. Van Gorder}

Mathematical Institute, University of Oxford, Andrew Wiles Building, Radcliffe Observatory Quarter,

Woodstock Road, Oxford OX2 6GG, UK

e-mail: Robert.Vangorder@maths.ox.ac.uk 
estimated experimentally and hence these expressions are not used here (they are simply taken to be fixed constants). We also introduce the parameter $\chi=\left(T_{0}-T_{\mathrm{S}}\right) / \Delta T$.

Unfortunately, there were typographical errors in two of the non-dimensional parameters in [1]. The correct nondimensionalization is $y^{*}=y / d, u^{*}=u / \tilde{u}$ and $\theta^{*}=\tau / \Delta T$, where $\Delta T=k \mu /\left(\rho^{2} \beta_{0}^{2} g^{2} d^{4}\right)$ and $\tilde{u}=\sqrt{k \Delta T / \mu}$. Note that this corrects the form of $\tilde{u}$. Here we omitted a factor of $\chi$ that canceled from $\tilde{u}$ and hence was not needed in the definition of $u^{*}$ in [1], and we have taken the appropriate scaling with $k$ rather than $K$ or $\chi$ as was incorrectly used in previous papers. These put (2.7), (2.11) and relevant boundary conditions into the form (dropping stars for convenience) of (2.12)-(2.14):

$$
\begin{aligned}
& \frac{\mathrm{d}^{2} u}{\mathrm{~d} y^{2}}-\frac{1}{\mathrm{Da}} u+\theta+\Gamma \theta^{2}=0, \quad \frac{\mathrm{d}^{2} \theta}{\mathrm{d} y^{2}}+\left(\frac{\mathrm{d} u}{\mathrm{~d} y}\right)^{2}+\alpha \chi=0, \\
& u(0)=0, \quad u(1)=0, \quad \theta(0)=\chi, \quad \theta(1)=m \chi,
\end{aligned}
$$

where $\mathrm{Da}=K / d^{2}$ is the Darcy number, $\Gamma=\Delta T \beta_{1} / \beta_{0}$ is the QDT parameter, $\alpha=Q^{\prime \prime} d^{2} /\left(k\left(T_{0}-T_{1}\right)\right)$ is the heat source/sink parameter, and $m=\left(T_{1}-T_{\mathrm{S}}\right) /\left(T_{0}-T_{1}\right)$ is the wall temperature ratio parameter. Note that this corrects the value of $\alpha$ which was incorrectly written as $\alpha=Q^{\prime \prime} d^{2} /\left(\chi\left(T_{0}-T_{1}\right)\right)$ in [1].

Since only non-dimensional parameters were used in the remainder of [1], the later sections and findings of [1] remain unchanged by the corrected scalings.

We wish to thank Professor A. Pantokratoras (Democritus University of Thrace, Greece) for bringing these typographical errors to our attention.

\section{References}

1. Akyildiz FT, Siginer DA, Vajravelu K, Van Gorder RA (2012) Natural convection heat transfer of a viscous fluid in a vertical porous channel. J Eng Math 74:61-71

2. Ostrach S (1952) Laminar natural convection flow and heat transfer of fluids with and without heat sources in channels with constant wall temperature. National Advisory Committee for Aeronautics, Technical Note 2863

3. Ostrach S (1954) Combined natural and forced convection laminar flow and heat transfer of fluids with and without sources on channels with linearly varying wall temperature. National Advisory Committee for Aeronautics, Technical Note 3141

4. Vajravelu K, Mohapatra RN, Soewono E (1993) Some second order nonlinear systems arising in natural convection heat transfer. J Math Anal Appl 176:346-358 